\title{
EL CUERPO COMO UNIDAD BIOLÓGICA Y SOCIAL: una premisa para la salud sexual y reproductiva
}

\author{
Giovanna Liset Reyes SÁNCHEZ \\ Médica de la Universidad del Valle, Magíster en Estudios Amazónicos \\ Universidad NaCional de Colombia \\ gioliset78@yahoo.com
}

\begin{abstract}
Resumen
[N ESTE ARTÍCULO SE PRESENTA UNA REFLEXIÓN SOBRE LA CONSTRUCCIÓN DEL CUERPO HUMANO EN [relación con la salud sexual y reproductiva. Plantea una discusión epistemológica acerca de la importancia que tiene para la medicina de hoy entender de una forma no reduccionista ni homogénea al cuerpo, con el objetivo de renovar, en el mediano plazo, las intervenciones en salud para que estén más acordes con los valores culturales y las características ambientales de los territorios que ocupan los grupos sociales que se pretenden beneficiar con las políticas de salud sexual y reproductiva. Hace referencia a la concepción y manejo del cuerpo entre los indígenas ticuna de la Amazonia colombiana.

Palabras ClaVE: cuerpo, salud, sexualidad, reproducción, salud pública, Amazonia.
\end{abstract}

\section{The bOdy as BIOlOgical AND SOCIAL UNITY:}

\section{A PREMISE FOR SEXUAL AND REPRODUCTIVE HEALTH}

\begin{abstract}
THIS ARTICLE REFLECTS ON THE CONSTRUCTION OF THE HUMAN BODY WITH RESPECT TO SEXUAL AND reproductive health. It presents an epistemological discussion on the importance for today's medicine to understand the body from a non reductionist or homogeneous perspective, in order to formulate renewed health interventions in agreement with the cultural values and environmental conditions of the territories inhabited by the beneficiaries of sexual and reproductive health policies. The article refers to indigenous Tikuna concepts of the body and body practices in the Colombian Amazon.

KEY WORDS: body, health, sexuality, reproduction, public health, Amazonia
\end{abstract}

Revista Colombiana de Antropología

Volumen 45 (I), enero-junio 2009, pp. 203-224 
El cuerpo como unidad biológica y social: Una premisa para la salud sexual y reproductiva

\section{INTRODUCCIÓN}

 N ESTE ARTÍCULO SE MUESTRA EL MOLDEAMIENTO DEL CUERPO HUMANO como un elemento central de la cultura, que nace de los grupos sociales y que puede afectar positivamente a los servicios de salud en Colombia en materia de políticas de salud sexual y reproductiva que pretenden cumplir con algunos de los Objetivos de Desarrollo del Milenio de la Organización de las Naciones Unidas, ONU, para el año $2015 .{ }^{1} \mathrm{El}$ cuerpo se propone como elemento central de la discusión con la intención de reencontrar el sentido del mismo para la práctica médica de los servicios de salud sexual y reproductiva, enmarcada actualmente dentro

I. Ver www.un.org

2. Ver www.colombia.unfpa.org (UNFPA-Colombia), www.col.ops-oms.org (OMS, OPSColombia), Plan Nacional de Salud Pública, objetivo 2, Decreto 3039 de agosto de 2007, Colombia. de los lineamientos de la ONU, la Organización Mundial de la Salud, OMS, la Organización Panamericana de la Salud, OPS, y el Sistema de Seguridad Social en Salud Colombiano. ${ }^{2}$

Los planteamientos actuales de las políticas en esta materia se han desarrollado a través de la historia conjuntamente con la medicina de occidente y demás disciplinas de la salud, como parte de la organización industrial del mundo moderno. De este proceso han surgido las intervenciones en este tópico, a partir del control de los cuerpos, primordialmente del femenino en la reproducción. De hecho, con el desarrollo de la ginecología y la obstetricia, el cuerpo de la mujer se ha confinado a la reproducción biológica y sobre él se ejecutan las acciones de los servicios de salud sexual y reproductiva, mientras que el cuerpo del hombre se ha construido en torno a la experimentación de la sexualidad. Desde la década de los 60 se ha hablado de las enfermedades propias de la mujer y del hombre, orientadas hacia los dos aspectos mencionados, con lo que se ha contribuido a desarrollos diferenciados de la industria farmacéutica en salud sexual y reproductiva. La mayoría de métodos anticonceptivos son para mujeres y los medicamentos para potenciar la sexualidad para los hombres.

En este sentido, se puede mencionar que la atención del parto que se realiza en Colombia, en la mayoría de instituciones de salud en la clásica posición de litotomía, consiste en que la mujer adopta una posición horizontal, con el pecho hacia arriba y las 
Volumen 45 (I), enero-junio 2009

piernas levantadas, colocándose en una silla especial para este fin. Esta posición fue introducida a la práctica médica obstétrica, en el siglo XVII en Europa, cuando comenzó el uso del fórceps para hacer más fácil el procedimiento de los médicos y no la comodidad de la mujer; de pensarse así el parto continuaría siendo en posición vertical. El discurso médico de occidente, muy difundido en contextos urbanos, utiliza un lenguaje masculino y militar sobre el cuerpo, basado en el modelo de la sociedad industrial. "El cuerpo como máquina", como un sistema donde cada una de sus partes trabaja para contribuir a la estructura general, comandadas por el sistema nervioso central mientras se defiende de agresores externos. Inclusive en los textos científicos se usan términos como: coordinación, regulación, respuesta a comandos u órdenes, envío de mensajes, procesos, funciones, entradas, salidas. Es decir, la "metáfora del cuerpo humano como máquina” está ligada a la ideología de la organización social industrial del mundo moderno (Martin, I992). Pero, lo que se ha logrado es la fragmentación del conocimiento médico, reflejada en su quehacer, en el estudio del cuerpo humano dividido en partes y de los determinantes de la salud y la enfermedad como variables.

Así, la construcción del conocimiento y la práctica médica en salud sexual y reproductiva se han orientado a definir la forma y el comportamiento de los cuerpos masculinos y femeninos para lograr los objetivos de salud pública: control de la natalidad, disminución de la morbilidad y mortalidad de madres y niños y de las enfermedades de transmisión sexual en hombres y mujeres, bajo el concepto de cuerpos económicamente productivos. El cuerpo es asumido e interpretado desde su funcionamiento biológico como eje central de la práctica médica, incluso al servicio de la industria farmacéutica, en tanto el conocimiento alopático se ha construido como una disciplina positivista, donde todos los fenómenos corporales deben ser demostrados por métodos científicos. Este conocimiento legitimado por la ciencia ha permitido ejercer el poder de guiar el comportamiento de los cuerpos, en especial, en las culturas que han apropiado las prácticas médicas de occidente.

Este artículo pretende mostrar, sin embargo, que este enfoque no logra ser efectivo para resolver las problemáticas de salud sexual y reproductiva debido a su visión reduccionista y homogénea sobre el cuerpo, a pesar de la mejora en coberturas de 
salud y las tecnologías biomédicas. Para demostrar lo anterior se parte por definir el cuerpo humano como una unidad que interconecta el "yo corporal” (Maturana, 1993) con otros cuerpos y sustancias. Es decir, es biológico y social de forma simultánea y esta unidad es construida por la cultura. En este sentido, presenta las características de los fenómenos culturales y es relativo a los grupos sociales y a sus experiencias personales. Por supuesto, esto va ligado a la construcción de los programas de salud sexual y reproductiva que deben considerar esta heterogeneidad de los cuerpos.

En este artículo, entonces, se propone un encuentro de la medicina con las ciencias sociales que aporte una definición del cuerpo más integral para las políticas de salud sexual y reproductiva, en este caso en Colombia. Se usan los resultados etnográficos de la autora en una comunidad indígena ticuna de la Amazonia colombiana (Reyes, 2008), aportes teóricos de las ciencias sociales y la discusión sobre la construcción y ejecución de las políticas de salud sexual y reproductiva en nuestro país, para construir argumentos que conducirán al lector por los diferentes conceptos y procesos que conforman al cuerpo humano: el "yo corporal", el cuerpo social, y el flujo de sustancias con los otros cuerpos y el territorio. Con el objeto de dar claridad conceptual se separarán estos procesos en tres partes si bien es difícil no tratarlos paralelamente.

\section{EL "YO CORPORAL" Y LA TRANSFORMACIÓN DEL CUERPO EN EL CICLO VITAL}

\footnotetext{
A EXPERIENCIA INDIVIDUAL DEL CUERPO TIENE LA MAYOR CARGA BIOLÓGICA DE todo este asunto. De una parte son inminentes las experiencias físicas y sensoriales vividas por cada cuerpo; de otra, nuestro cuerpo biológico es el eje central de la educación familiar, escolar y de los programas de salud sexual y reproductiva; es decir, el comportamiento biológico y la forma física de nuestros cuerpos son moldeados por la cultura. Desde niños se nos dice qué hacer, cómo mover el cuerpo, "siéntate así", "no hagas esto, hazlo así", "no brinques, no grites", "come esto o lo otro", "ganaste el examen, te invito un helado", "no abras la nevera acalorado", "ponte esta u otra vestimenta". Esto se puede comprender me-
} 
jor en el campo de la sexualidad. El ser humano es también un cuerpo que sostiene relaciones sexuales, pero aquí la biología no impone las reglas sobre con quién tener sexo, en qué momento, con qué finalidad, si es por placer, necesidad o coerción, de modo frecuente o esporádico, estimulando éste o aquél punto erógeno, en la fase inicial de la formación de una persona o como mecanismo de perduración como grupo (Rodrigues, 2005). Al contrario, es una construcción cultural. Con frecuencia se escuchan frases como: "no te toques", "debes empezar a tener sexo a tal edad", "no debes quedar en embarazo tan joven", "mueve tu cuerpo así, no lo muevas así", "las mujeres son delicadas", "los hombres son fuertes", "escoge a un buen hombre", "escoge a una buena mujer". La cultura moldea los cuerpos y de un repertorio de opciones, los hombres y las mujeres inventan sus cuerpos que son "construidos con adornos, tinturas y prótesis, con disciplina, trabajo físico y entrenamiento, con automatismos adaptados a los mobiliarios, a las herramientas y las máquinas, con placeres y sufrimientos corporales físicos, simbólicos e imaginarios" (Rodrigues, 2005: 179).

El "yo corporal” se puede definir como el territorio físico individual que ocupa cada persona. El cuerpo es la primera casa pero es también la forma que tiene, las sustancias que lo forman, cada uno de los órganos, músculos y movimientos, los cambios que se experimentan a lo largo del ciclo vital, la conexión entre cada una de las partes del cuerpo y de toda la unidad con otros cuerpos, dentro del territorio ambiental y social que ocupa. En el campo de la salud sexual y reproductiva, la adquisición de conciencia del cuerpo se propone como un reto, es una forma de obtener autoconocimiento y autonomía, dos elementos fundamentales de la educación y la promoción de la salud. También son el objetivo de la educación clásica en salud del cuerpo, que se concibe como receptor de información de los profesionales de la salud sin que necesariamente sea apropiada por parte de los cuerpos adoctrinados, ya que la mayoría de veces no se adecua a la realidad y a la experiencia corporal de cada persona o grupo social.

Hacer conciencia de la experiencia biológica y social del cuerpo a lo largo del ciclo vital es fundamental para la salud sexual y reproductiva de la población colombiana. Cada persona en su vida atraviesa por etapas de cambio y adaptación, que desde el 
punto de vista de la salud son: gestación, parto y nacimiento, puerperio, infancia, pubertad, menarquia, juventud, adultez, vejez y muerte. Al igual que el planeta tierra, las personas tienen un comportamiento cíclico de transformación, de vigilia y sueño, de luz y oscuridad, de flujo de sustancias: sangre, leche materna, alimentos, semen, orina, materia fecal, flujo vaginal, o sea, de aire, fuego, agua y tierra. Esta experiencia es vivida de manera explícita e intensa por cada cuerpo, aunque en la cultura de occidente influencias religiosas, médicas y culturales han negado el conocimiento y la vivencia individual corporal. La dualidad cuerpo-mente, asociada con el desarrollo del mundo moderno industrial, ha favorecido las funciones mentales a costa de la negación de las sensaciones, percepciones y sustancias. El conocimiento y manejo corporal se ha delegado a la institucionalidad médica, al punto que la conciencia del cuerpo sólo se adquiere cuando se presentan la enfermedad y el dolor.

En algunos grupos sociales, el control del umbral del dolor, de la respiración, de las posiciones del cuerpo así como los rituales de dieta y reclusión, representan prácticas de control de los procesos biológicos, pero a su vez permiten entender al "yo corporal" en relación con otros o con el territorio, es decir, para hacer conciencia del cuerpo físico. Este es el caso de los indígenas ticuna, cuya experiencia de manejo y regulación del cuerpo, ilustra la discusión planteada hasta ahora. En concreto me referiré al ritual de iniciación femenina (Reyes, 2008), con el fin de hacer visible una práctica de construcción y cuidado del cuerpo que tiene como centro de acción a la mujer, en una de las etapas del ciclo vital: el primer flujo menstrual. La menarquia da inicio a la realización de un ritual que limpia y protege tanto al cuerpo de la joven, que se transforma en mujer, como a su familia. El ritual es conocido como la fiesta de la pelazón (yu-u-echíga) y alude al momento central de la fiesta cuando se corta el cabello de la muchacha para convertirla en una "recién nacida" y simboliza el paso de la niña a una mujer madura.

Este es un momento de alegría por el cambio corporal de la joven, a quien debe conducirse por el camino correcto, por lo que debe prepararse y recibir los consejos de su familia para asumir y enfrentar esta transición. La joven en esta etapa es llamada en lengua ticuna wôrekü.

La pelazón comienza con el encierro de la joven; éste es primordial para protegerla pues, según la cosmología ticuna, el 
Volumen 45 (I), enero-junio 2009

cuerpo de la wôrekü es frágil y vulnerable hasta cuando se realiza la fiesta. El encierro semeja la formación de una crisálida que se guarda y espera su tiempo para transformarse y convertirse en un nuevo ser; representa la preparación para un nacimiento, como se muestra en los siguientes fragmentos de los consejos cantados por las abuelas a una wôrekü:

—Esta fiesta viene de nuestros ancestros. Actualmente la estamos narrando. Por eso te encerraron como un capullo de seda. Por ese motivo estás como una niña recién nacida. Llegó el momento de pintar a la muchacha —wôrekü - con achiote. Con plumas engalanaremos su cuerpo. Sus piernas emplumadas serán como las de una mariposa. Cuando salga todos verán sus adornos. Pronto saldrá a su encuentro. Su cuerpo está transformado como el del cucarrón escamado. Hemos esperado este momento, ansiosos. Sale a nuestro encuentro para que la admiremos. Saldrá de pie, dejará el nido, saltará como pez por las quebradas (Camacho, I995: 70).

Durante este ritual los parientes cercanos la esperan y la preparan para que sea fuerte y tenga un comportamiento socialmente aceptado, para ser una "persona verdadera”. Después de la pelazón, la joven nace en un nuevo ser, en una mujer madura, lo que significa que a la agencia ${ }^{3}$ de la mujer se le otorga una posición central en la vida social, como se verá con mayor profundidad más adelante. Por ahora, se describen algunos momentos de una fiesta de pelazón a la que asistí (Reyes, 2008: 163):

3. Agencia es un concepto traducido del inglés agency, que se refiere a las capacidades de acción que constituyen a la persona inscrita, hecha y actuante en un mundo de interacciones, de producción, depredación, intercambio y consumo (Belaunde, 2005:37).

El sonido de los tambores daba inicio a la celebración mientras la joven permanecía encerrada. Los parientes y amigos invitados arribaban paulatinamente. Cada uno llevaba su clan pintado con el azul intenso del huito, en la cara o alguna parte del cuerpo. A mí me correspondió el clan Vaca, que fue dibujado en mi hombro. Cuando llegaron todos los invitados, la niña fue retirada de su encierro y presentada a los asistentes, su cuerpo estaba negro, tenía puesta una corona de plumas que cubría sus ojos. Vestía una falda roja y sobre su pecho desnudo caían collares largos de chaquiras multicolores de donde colgaban, como dijes, varios loros pequeños disecados ubicados en media luna. Tenía plumas blancas pegadas con achiote en brazos y piernas y lucía manillas y tobilleras de chambira decoradas con semillas o plumas. Mientras era retirada del encierro y se dirigía hacia el centro del recinto, su hermano la guiaba, 
ubicado frente a ella, sujetaba con sus manos la corona de plumas en la cabeza de la joven. A su lado estaban su madre, su abuela y el médico tradicional que se encargaría de realizar la "curación de la joven”. Su tío fue designado por su padre, dueño de la fiesta, como encargado de supervisar todos los pasos del ritual.

La joven fue presentada a todos los invitados. Mientras se repartía masato, la guiaban por toda la casa hasta que la ubicaron en el centro donde esperaba su tío. Ahí comenzó un baile donde los invitados se desplazaban hacia delante y atrás al ritmo de los tambores y del bastón de mando del médico tradicional, que tenía sujetadas en la parte inferior unas semillas que producían un sonido particular. Mientras tanto la joven estaba en el centro del grupo con su hermano enfrente quien le cubría sus ojos con la corona de plumas. Después, fue sentada en el piso sobre una estera y a su lado se ubicaron su madre, su abuela, el dueño de la fiesta y el médico tradicional. Mientras tanto continuaban los tambores, el golpe del bastón de mando y el médico tradicional soplaba el cuerpo de la joven con tabaco.

En ese momento fueron ubicadas a su lado, con sus madres, varias niñas pequeñas con edades entre uno a tres años, que también fueron pintadas con huito y decoradas con plumas y collares como la joven de la pelazón. Estas pequeñas fueron sometidas a su primer rito de iniciación que consiste en abrir los orificios de las orejas mientras le cortan el cabello a la joven. Cuando la joven fue ubicada en el centro, su hermano retiró la corona de plumas por orden del médico tradicional y se procedió a realizar la pelazón, que en este caso estuvo a cargo de su madre y abuela. El médico tradicional soplaba tabaco sobre su cabeza y el resto del cuerpo y emitía cantos y rezos. El dueño de la fiesta, por su parte, golpeaba con el bastón y entonaba los cantos.

Luego vino el canto de las bocinas y de los tambores por parte de los familiares más cercanos que se acercaron a ella y comenzaron a bailar al ritmo de los instrumentos; las niñas pequeñas también participaron de este momento. Así, se formó un gran círculo humano que acompañaba y protegía a la joven.

El ritual de la pelazón culmina con el baño de la joven en la quebrada, lugar donde el médico tradicional sopla con tabaco a la joven para "curar su cuerpo": evitar que tenga hijos a temprana edad y convocar la abundancia de peces y la fertilidad de la tierra. Es decir, el ritual también le rinde tributo a la fertilidad y a la abundancia, por medio del cuerpo de la wôrekü.

Para diferentes pueblos indígenas amazónicos ciertas etapas del ciclo vital exigen realizar rituales de cuidado para mantener el cuerpo fuerte y sano, que sin considerarlas enfermedades en sí mismas exigen disciplina y conciencia del cuerpo. Los cuerpos femeninos y masculinos se forman durante las etapas de 
transformación corporal que se presentan a lo largo del ciclo de vida, mediante prácticas tan cotidianas como la alimentación y ritos de dieta y reclusión. ${ }^{4}$ Éstas son realizadas para dar fuerza y evitar enfermedades en momentos de transición a otro estado físico y espiritual, que implican una transformación corporal y una vulnerabilidad de la persona en sí misma o en su relación con el territorio. En el caso de la pelazón, el encierro constituye un espacio de reflexión y disciplina inicial sobre el cuerpo donde la joven, entre otras, debe adquirir habilidades para el tejido de la chambira ${ }^{5}$, e independiente del número de piezas que procese, con esta labor la joven debe demostrar disciplina corporal y elementos fundamentales para convertirse en una mujer madura o "mujer que sabe".

Lo anterior es un ejemplo de cómo los grupos sociales construyen sus cuerpos a través de la cultura y de cómo la experiencia corporal individual se entiende y asume de maneras diferentes según las concepciones y los valores del grupo social. De acuerdo 4. La conciencia del cuerpo se adquiere mediante prácticas como guardar ayuno o comer sólo un tipo de alimentos, someterlo a un periodo de encierro que se realiza en la casa familiar, en la maloka (vivienda tradicional indígena) o en la selva. Según la etnia, esto sucede durante el periodo menstrual y el posparto. El encierro también es una práctica durante la preparación de los hombres como chamanes. Estas son formas de limpiar el cuerpo, conservar la fuerza vital y evitar la enfermedad.

5. Fibra de la palma Astrocaryum chambira, usada para realizar tejidos, actividad artesanal y artística practicada por las mujeres ticuna. con la experiencia de los ticuna y al resumir la perspectiva de Rodrigues (2005), se puede afirmar que el cuerpo es mucho menos biológico de lo que se pensaba, sin negar esta dimensión. Aunque la medicina de occidente haya pregonado que los cuerpos se comportan biológicamente bajo un patrón regular y que "su dimensión central no es precisamente el bienestar, no son sólo las personas, ni siquiera sus cuerpos, pero sí sus cuerpos durante la enfermedad" (Hanh y Kleinman, 1983: 3I2), la realidad es que las personas más que una máquina son un cuerpo construido socialmente; por lo tanto, la consideración de las diferencias étnicas, ambientales, de género o socioeconómicas son decisivas en la formulación y ejecución de intervenciones en salud sexual y reproductiva.

Aquí reside el asunto central del "yo corporal”, tan relevante en estos tiempos para las políticas de salud sexual y reproductiva. Si cada persona conoce su cuerpo y sabe quién es como mujer y hombre, como parte de un grupo social y un territorio, puede tener autoconocimiento y la suficiente autonomía para tomar 
decisiones acertadas en la vida. Pero esto no depende de las instituciones sino del contexto social, de la educación familiar y escolar y de un enfoque adecuado de la educación y promoción de la salud. El aseguramiento universal, los subsidios en salud, los programas de apoyo a las familias a través de métodos asistencialistas o el fácil acceso a métodos anticonceptivos, sin una adecuada apropiación de las personas de sí mismas y de sus cuerpos, de acuerdo con sus propios valores culturales, no harán posible en el largo plazo que la población colombiana construya una vida individual y social plena, donde se incluya el libre desarrollo de la sexualidad y la reproducción, sin formas de coerción y violencia.

\section{EL CUERPO: UNA CONSTRUCGIÓN SOCIAL}

EGÚN RODRIGUES (2005), LAS SOCIEDADES CONSTRUYEN SUS CUERPOS,
pues una sociedad se forja haciendo los cuerpos en los que
existe. La construcción de la persona o del cuerpo como concepto de las ciencias sociales fue mencionada por los antropólogos Seeger, Da Matta y Viveros de Castro (1987) con base en su trabajo con grupos indígenas amazónicos. Seeger lo planteó con estos términos: "El cuerpo, afirmado o negado, pintado o perforado, recluido o devorado, tiende siempre a ocupar una posición central en la visión que las sociedades indígenas tienen de la naturaleza del ser humano”. Según la visión que se tenga sobre el ser humano se construyen las sociedades y en ese mismo sentido es construido el cuerpo. En las sociedades industriales modernas los cuerpos son fuerza de trabajo y producción para las industrias; se promueve la individualidad en lugar de lo colectivo, la estética en lugar de la salud, la homogeneidad en lugar de la diferencia; los cuerpos producen y acumulan bienes y servicios, en vez de alimentos; se controla la natalidad para disminuir la pobreza, medida en ingresos económicos. En las sociedades indígenas los cuerpos también son fuerza de trabajo pero para el beneficio del grupo social; están interconectados con el territorio que los rodea: la selva o la montaña. Hay una promoción de redes sociales entre parientes o con otros grupos sociales; un cuerpo fuerte es saludable más que estético; los cuerpos producen alimentos en lugar de bienes y servicios y los cuerpos se reproducen biológica 
Volumen 45 (I), enero-junio 2009

y socialmente en otros cuerpos; se controla la natalidad para tener un acceso adecuado a los recursos del territorio.

Belaunde (2005), en su libro El recuerdo de Luna: género, sangre y memoria entre los pueblos amazónicos, realiza un análisis de varias etnografías amazónicas y confirma que para los pueblos indígenas la corporalidad no se fragmenta de otras dimensiones de la vida social. Por el contrario, muchos aspectos de la cotidianidad se disponen a partir de la construcción del cuerpo como mecanismo de socialización de hombres y mujeres. Son muchos los estudios etnográficos realizados con pueblos indígenas amazónicos (McCallum, I996; Perrín y Perruchón, i997; Overing y Passes, 2000; Mahecha, 2004; Londoño, 2004; Belaunde, 2005; Nieto, 2006; Reyes, 2008) que han tratado el concepto de agencia como una forma de expresión de la corporalidad en la vida social, en la formación de redes de parentesco y solidaridad y en la construcción de otros cuerpos masculinos y femeninos con el fin de formar "personas verdaderas". Este concepto se inscribe en el sentido moral de ser una persona con una conducta aprobada por la sociedad (Mahecha, 2004) y varía para cada etnia. Para los macuna por ejemplo, la capacidad de concebir, cuidar y criar los hijos es virtud de "personas verdaderas" (Mahecha, 2004: 173). En el caso de una comunidad indígena ticuna cercana a Leticia ${ }^{6}$ (Reyes, 2008), una de las abuelas considera que si una mujer no conforma una familia:

-No es como uno que lleva buen camino, se hace anciano. Se es anciano, se tiene nietos, los nietos tienen esposos y ellos viven como uno. Viven bien, en ningún momento lloran, en ningún momento se pelean, se tiene nieto, tienen esposos y ellos cogen nuestro camino y viven como nosotros. Ellos crecen aquí, es bueno aconsejarlo para que cuide su cuerpo, pero cuando se dice así [a los hijos], se enojan. A nuestras hijas les debemos aconsejar. Su mamá también le aconseja al hombre. Porque es la mujer que debe quedar ${ }^{7}$ y se le debe entregar de una vez. Se les hace casar frente al sacerdote. Y como dicen antiguamente: "Ahí está y a enfermarse con ella". Eso es cierto, mire mis hijas que tienen marido, en ese tiempo su abuelo va donde ella, va y la aconseja - ahora que van a tener marido no debe abandonarlo, no debe mirar a otro hombre. Mientras tanto su madre

6. Capital del departamento del Amazonas, Colombia.

7. Patrón de residencia uxorilocal o matrilocal de los ticuna. La mujer permanece en su comunidad y el esposo se traslada al hogar de ella. también aconseja al hombre. Es el único con quien debes vivir para siempre. No quiero otra gente, no quiero gente blanca porque no 
sabe hacer nada, yo quiero es gente como nosotros, es aquel que sabe pescar, hacer canoa, hacer canoa contigo.

Las palabras de aprobación moral de la abuela expresan que quien sigue el buen camino se hace anciano y tiene hijos y nietos a quienes da consejos y enseña a cuidar su cuerpo y a trabajar; que el buen esposo por lo usual está relacionado con el buen trabajador. Saber cultivar los alimentos, construir canoa, casa y mantener una familia junto a su mujer, revelan la manera como se busca formar personas, que con su buen comportamiento y con cuerpos fuertes le aporten no sólo a la familia sino al grupo social. Representan la construcción social del cuerpo para los ticuna. Con base en la experiencia ticuna, es posible afirmar que el cuerpo no sólo es menos individual e individualizado de lo que se acostumbra postular en el pensamiento biomédico, sino que es construido socialmente (Rodrigues, 2005). En esta comunidad indígena, el cuerpo es parte de otros y se construye por acción de ellos; significa que la vida se protege desde la gestación y se forma por los cuidados que otros tienen de ella en un proceso que moldea los cuerpos desde la concepción hasta la muerte. El ritual de la pelazón, organizado con disciplina por la red de parientes, conduce a la niña por el camino que la lleva a ser una mujer madura o "que sabe". La transición corporal desencadena un ritual de protección y renovación de energías con la tierra que moldea el cuerpo de la joven durante el encierro, tal como la crisálida que luego se convierte en mariposa. Además, durante el ritual los parientes adquieren conciencia del territorio y de la red social de la que hacen parte.

La fiesta de la pelazón representa una práctica de la vida cotidiana que promueve la salud, previene la enfermedad y que tiene un impacto importante para la salud sexual y reproductiva dado que pretende, entre otros aspectos, evitar gestaciones no planeadas mediante la preparación de la joven y su cuerpo, para entender su posición en la vida social comunitaria, para ser fuerte en el trabajo y la reproducción y para su desempeño en la vida, en la pareja y en la familia. En el proceso de construcción como persona, como "mujer que sabe", la joven enfrentará la reproducción social pues tendrá la responsabilidad de formar otros cuerpos; pero antes, el suyo debe adquirir madurez física, razón por la que durante la fiesta el médico tradicional realiza 
su "curación", con lo que contribuye a la regulación de la fecundidad. De esta forma, ella puede esperar algunos años para comenzar a tener hijos, mientras su cuerpo se fortalece a través del trabajo. En otras palabras, cuidar el cuerpo de las jóvenes redunda en la salud de sus parientes y su descendencia, tiene que ver con el mantenimiento de los lazos de parentesco, la abundancia de alimentos, la fertilidad de la tierra y la conservación del territorio. Es una afirmación del cuerpo individual, social y del gran cuerpo que es la tierra. Asimismo, al ser el cuerpo, en gran medida, una construcción social, en el se reproducen los valores culturales del grupo social, es dinámico y heterogéneo, como la cultura misma.

En la construcción del cuerpo intervienen los padres, los parientes, la comunidad, los profesores en la escuela, los amigos y la pareja. Estos son referencias claves para la vida individual y social en tanto la educación para la vida no depende sólo de la intervención de las instituciones de salud, sino del grupo social y de la posibilidad que tenga una comunidad de desarrollar una vida social y productiva plena, según sus propios valores culturales, en donde se pueda decidir con libertad la escogencia de la pareja, el número de hijos, el acceso a los recursos del territorio y a los servicios de salud, culturalmente adecuados a las necesidades locales. Esta dimensión es fundamental para la promoción de la salud sexual y reproductiva porque al tener primero control sobre el "yo corporal", la persona puede formar otros cuerpos y poner parte de sus sustancias y consejos en otro.

De acuerdo con lo anterior, resultará difícil obtener resultados positivos en relación con los Objetivos de Desarrollo del Milenio, a partir de programas de salud sexual y reproductiva que pretenden, mediante charlas o talleres sobre autonomía y derechos sexuales y reproductivos, generar un cambio en las concepciones y los comportamientos si los contenidos y los métodos empleados no se adecuan a los valores culturales del grupo social, y si las condiciones sociales y económicas de las poblaciones no permiten el desarrollo de una vida social plena. 
El cuerpo como unidad biológica y social: Una premisa para la salud sexual y reproductiva

\section{El flujo de sustancias}

L CUERPO EXPRESA LO QUE SOMOS, LO QUE COMEMOS, DÓNDE, CON QUIÉN Y
cómo vivimos, nuestro trabajo y emociones. El moldeamiento
social de los cuerpos se da desde la gestación hasta la muerte
pues la reproducción no sólo es el acto de concebir y dar a luz
una nueva vida, sino formarla a lo largo del ciclo vital. Según
Viveros de Castro, de acuerdo con su experiencia amazónica:

El cuerpo humano necesita ser sometido a procesos intencionales y periódicos de fabricación, entre los cuales las relaciones sexuales entre los progenitores de un futuro individuo constituyen apenas el momento inicial. Tal fabricación es concebida, aunque no exclusivamente, como un conjunto de intervenciones sobre las sustancias que comunican el cuerpo humano y el mundo: los fluidos corporales, los alimentos, el tabaco, los aceites y las tinturas vegetales. Por esta razón, en estos grupos sociales no es posible la distinción ontológica entre procesos fisiológicos y procesos sociológicos, debido a que las transformaciones del cuerpo y de la posición social son la misma cosa. Es decir, lo social no se deposita sobre el cuerpo como un soporte inerte, sino que forma a ese cuerpo. ${ }^{8}$

Volviendo al caso de la pelazón, se considera éste un momento donde la joven recibe consejos y cuidados. Cada vez que tiene la menstruación debe bañarse con una cocción de plantas medicinales como la "hoja de algodón” y la "cáscara del árbol de uvo" (yómeru), y no consumir alimentos que aumenten el sangrado y conlleven la aparición de cólico menstrual, entre ellos pescados como la piraña y la "cucha" o las frutas ácidas. Debe también evitar el contacto con personas diferentes a la familia con la que convive bajo un mismo techo, sólo su madre o su abuela le llevan el agua para el baño diario y la comida y nunca debe bañarse con agua fría, siempre tibia. Durante este periodo de flujo de sangre se debe proteger a la joven de complicaciones asociadas, entre ellas, evitar el frío corporal pues está relacionado con el aumento

8. Viveros de Castro, I979: I. (Traducción libre de la autora.)

9. El calor y el frío en relación con la presencia de enfermedades es transversal a muchas culturas, lo que ha sido muy discutido por la antropología médica. Su relevancia en este artículo radica en su relación con el flujo de sustancias del cuerpo con el exterior. de hemorragias uterinas, cólicos y pérdida de fuerza. ${ }^{9}$

El ritual conlleva también un flujo de entrega y recepción de sustancias como sangre, plantas y alimentos que se intercambian con los parientes o los "dueños" 
de la selva (ngo-ogü). ${ }^{10}$ Las sustancias forman, cuidan y acompañan al cuerpo y sus procesos, pero en esta etapa de transformación se requiere que no haya enfermedad para realizar el ritual, por lo tanto el cuerpo debe estar en equilibrio consigo mismo, con los otros, con el territorio y con los seres no humanos de la selva, los inmortales. Dos mujeres ticuna describen algunos cuidados:

- Cuando es señorita se la guarda en toldillo para que no salga a ningún lado; no debe ir a ningún lado por la gente, porque si la gente la ve, ella tendrá más dificultades. Como hoy en día es otro momento, está bien hacer la celebración. Y cuando le llega la menstruación hay que darle remedio, hacerle remedio. Se cocina corteza de uvo y se hace de todo (Relato de Pascuala Fernández, San Sebastián, marzo de 2007).

-Apenas que se desarrolla, la guardan en la cama, allá donde nadie la ve, solamente la familia; cuando llega visita ella se esconde. Entonces si el hermano [la ve] se vuelve salado pues el mundo, el medio ambiente, se contamina, no da buena protección. Todo eso [la sangre que sale] afecta el mundo que nos rodea, porque parece que cuando uno [la mujer] está menstruada, toda esa sangre que uno bota, pues, uno perjudica a toda la naturaleza (Relato de Ruth Lorenzo, San Sebastián, marzo de 2007).

El flujo de sangre de la wôre$k \ddot{~ t r a e ~ e l ~ r e c u e r d o ~ d e l ~ o r i g e n, ~}{ }^{11}$ de la necesidad de limpieza e intercambio espiritual con los "dueños" de la selva, y de que lo que acontece en un cuerpo puede afectar a los demás. Durante la fiesta de la pelazón los mitos de origen vienen y van a través de los cantos, que cumplen la función de mantener viva la memoria del origen de su etnia y sus ritos, reafirmar la agencia de la mujer en la vida social, mantener la armonía con la naturaleza y limpiar sus cuerpos. El ritual de la pelazón sigue vigente en la cultura ticuna porque devuelve el recuerdo del origen en el cuerpo de la wôrekü; cada vez que menstrúa una wôrekü, es como si lo hiciera la madre tierra.
IO. La cosmología ticuna considera que en la organización del mundo hay una división entre seres mortales e inmortales. Los primeros son los humanos, animales y plantas y los segundos los dueños espirituales de los animales y plantas, denominados ngo-ogü. Dentro de este grupo también se encuentran los seres pescados por los dioses originales Yoí e Ipi, considerados sus ancestros y denominados "los antiguos". Para los ticuna el universo se mueve en un conflicto constante entre mortales e inmortales, por lo que debe existir un flujo de energía que mantenga la armonía entre estos dos mundos. De esto depende que se mantenga la salud y no haya enfermedad entre los humanos. También consideran que todos los seres tienen porá que es la energía, la fuerza de la que están provistos y cuya acumulación les permite sobrevivir a los ataques de los ngo-ogü y evitar enfermedades (Goulard, 1994, 2006c; Reyes, 2008).

II. La menstruación de la wôrekü revive la situación del incesto entre Ipi y Mowacha como lo cuenta su mito de origen. 
Otra manifestación del flujo de sustancias que moldea el cuerpo en este ritual es la decoración del cuerpo de la wôrekü. El día anterior a la llegada de los invitados, la joven es pintada y decorada con cuidado por su madre, abuelas y tías en el "corral" dispuesto para este fin, y que los hombres construyen con la corteza de la palma de canangucho o aguaje. Todo su cuerpo es impregnado con tintura de huito ${ }^{12}$, que al secarse sobre la piel da un color azul índigo tan oscuro como el negro. Una abuela de la comunidad explicó la razón de esta práctica:

-Eso es así, el huito es para pintarse el cuerpo. Eso viene desde antes, antes cuando mi finada mamá contaba. El huito sirve para que los "yureos" [ngo-ogü] no se les acerque, porque el cuerpo huele a huito, para eso es que se pinta el cuerpo (Relato de Hilda Lorenzo, San Sebastián, marzo de 2007).

En resumen, el cuerpo de la wôrekü debe protegerse con el encierro y la dieta, debe semejar un cambio de piel y no debe oler a sangre; por eso se pinta y transforma su piel con huito y achiote ${ }^{13} \mathrm{y}$ al final se corta el cabello. Los parientes también deben

I2. El huito es el fruto del árbol Genipa americana. Se ralla y exprime antes de madurar para obtener su jugo del que se prepara un tinte de color azul índigo intenso y oscuro. Los ticuna lo usan sobre la piel en especial en el ritual de iniciación femenina.

I3. Semilla del árbol Bixa orellana que al macerarse produce un color rojo naranja.

I4. El clan es el primer nombre que se le da a una persona después de nacer, el que lo hace pertenecer a una familia y define su consanguinidad. Los clanes se heredan de forma patrilineal, así que los hijos pertenecen a una de las mitades clánicas: los de pluma (paujíl, páucara, guacamaya, gallina) o sin pluma (tigre, hormiga arriera, cascabel). Cada miembro de una pareja (mujer y hombre) debe pertenecer a una mitad clánica, como lo menciona Hernando Lorenzo. "Lo que tiene la muchacha [el clan], [es] lo contrario de su marido, ahí pueden vivir" (San Sebastián, marzo, 2007). llegar a la fiesta con su rostro pintado con el distintivo del clan ${ }^{14}$ al que pertenecen. Deben además proteger y dar fuerza al cuerpo de la joven, con los alimentos adecuados, aconsejarlo, tocando los tambores y haciendo sonar las bocinas durante la fiesta para anunciar a los inmortales que ellos persisten como mortales y que protegen a la muchacha de la enfermedad y del encantamiento producidos por espíritus malignos de la selva (ngo-ogü), también llamados "yureos", "diablos" o "demonios". En palabras de una abuela,

—Los demonios no matan cuando uno se hace pelazón; el demonio nos busca y cuando ya se hace pelazón, el demonio no pasa, no más porque nosotros olemos como ellos. Cuando no se hace pelazón nos busca, nos coge y nos parte por la mitad, nos huele. Y cuando uno se sale de la pelazón no huele, no huele y uno se va de una vez a la 
casa y le dice: "Usted ya salió de pelazón, por eso usted no huele" (Relato abuela Pascuala, San Sebastián, marzo de 2007).

Desde la preparación y durante la realización de la fiesta, los lazos de parentesco se fortalecen. Vienen parientes de sitios lejanos y todos participan de una u otra forma. Este evento construye una "consustancialidad" social, pues se comparten alimentos y bebidas, todos se entrelazan y bailan al ritmo de los tambores formando un tejido humano de protección alrededor de la wôrekü. La "consustancialidad" es el hecho de que el cuerpo se forma por las sustancias que se reciben de otros: la sangre, el semen, la leche materna, el masato, la fariña y, en general, los alimentos. El parentesco es una cuestión de "consustancialidad" y si los padres asumen cuidados con sus cuerpos, a su vez protegen a sus hijos y evitan la enfermedad, pues los hijos están formados por sus propias sustancias.

Como sucede en otros grupos étnicos, el control del flujo de recepción y entrega de sustancias del cuerpo tiene como fin fundamental moldearlo pero a la vez establecer un flujo controlado de energía con el territorio, que implica la conservación de la fuerza corporal y la prevención de enfermedades. Las sustancias que el cuerpo recibe, como los alimentos, participan en la acción de "consustancialidad" con otros cuerpos como las plantas o animales, los parientes más cercanos o el hijo que se lleva en el vientre. Los alimentos son sustancias transformadas, por lo usual por las mujeres, que se dan a consumir a la familia y de forma simultánea construyen el cuerpo. Durante la gestación este aporte es más contundente porque los alimentos colaboran con las sustancias de los padres, que se han mezclado para formar una nueva vida sin enfermedades.

Los ticuna consideran que las sustancias de ciertos animales, que están cargados de mucha energía (porá) como: serpientes, grandes peces de cuero o escamas, gusanos como el mojojoy y otros animales de monte, pueden enfermar a las personas. El olor de la sangre hace visibles a los humanos frente a los espíritus de estos animales y por eso se pinta el cuerpo de la joven menstruante con huito para que el cuerpo no huela. Por esta razón, la puérpera y el recién nacido deben recluirse también. La protección de la joven contra la enfermedad implica la regulación del flujo de energía con el territorio. El flujo de sustancias dentro de cada cuerpo, de este hacia el exterior y en sentido contrario, recuerda 
que en materia de salud no se debe tener una visión antropocéntrica del asunto. La relación e interconexión con el territorio es primordial para la supervivencia de los seres humanos. En cuanto a la salud sexual y reproductiva, dicha conexión es clave porque la formación y organización de las familias, de la red de parientes y de la vida productiva dependen de la ubicación de un grupo social en un territorio. Sin él, se quedan sin sustento las políticas de salud pública que pretenden disminuir la pobreza y mejorar las condiciones de vida, incluidas el libre desarrollo de la sexualidad y la reproducción biológica y social.

Los programas de salud sexual y reproductiva orientados a promover la lactancia materna, la "alimentación balanceada" en los menores de cinco años y las gestantes, el uso de métodos anticonceptivos y la prevención de enfermedades de transmisión sexual, se pueden dirigir de forma adecuada si se comprende la relación de los cuerpos con las sustancias de su propio cuerpo, de los otros y del territorio; si consideran los valores culturales de los grupos sociales y las características ambientales de sus territorios y no sólo las prácticas determinadas por la medicina o la nutrición de la cultura de occidente.

\section{A MANERA DE CONCLUSIÓN: \\ TRES PREMISAS SOBRE EL CUERPO \\ PARA LA SALUD} N DEFINITIVA, EL CUERPO ES LA CONJUNCIÓN DE TRES PREMISAS: LA
primera es la conciencia del “yo corporal”, la segunda es su
naturaleza socialmente construida y que encarna la dinámica
de la cultura, la tercera es que está conectado con otros cuerpos
y sustancias. Una sociedad se conforma haciendo los cuerpos
en los que ella existe.

Asimismo, la salud sexual y reproductiva es salud, es "vivir bien" (Belaunde, 2005), procurar mantener el cuerpo fuerte y sano; moldear el propio cuerpo y los cuerpos de los parientes mediante la educación; hacer conciencia del cuerpo y sus transformaciones; construir una familia y una red social de protección y solidaridad con los parientes y mantener un flujo equilibrado de sustancias y energía con los otros y con el territorio. Por estas razones, la promoción de la salud y la prevención de la enfermedad no se 
pueden fragmentar en áreas determinantes o en partes del cuerpo y del ciclo vital, ya que las prácticas sociales para "vivir bien" son construidas por cuerpos femeninos y masculinos como una unidad, en interacción con otros y con el territorio.

El planteamiento de una forma integral de entender y abordar al cuerpo humano, en relación con la salud sexual y reproductiva, busca refrescar con nuevos conocimientos la educación médica convencional, que prepara profesionales para intervenir sobre un cuerpo fragmentado y elabora algoritmos sobre los tratamientos de las enfermedades con la pretensión de actuar sobre cuerpos homogéneos haciendo uso, en la mayoría de los casos, de fármacos que estabilizan ésta o aquélla función biológica. Y aunque estas intervenciones son efectivas para resolver problemas cotidianos de salud o para algunas enfermedades específicas a nivel individual, estamos ante una profusa cascada de problemas de la salud sexual y reproductiva y de los cuerpos femeninos y masculinos tales como: cáncer, enfermedades de transmisión sexual, trastornos de la alimentación y del desarrollo de la sexualidad, mortalidad infantil y materna, diversas formas de violencia y discapacidad. Todos estos males se presentan en cuerpos heterogéneos, biológica y socialmente, por múltiples causas y no logran ser controladas sólo con fármacos, ni siquiera con mayores coberturas del sistema de salud o con las mejores tecnologías biomédicas. El camino que se muestra para la medicina en los tiempos actuales de cambio climático, tensiones sociales, guerras y enfermedades emergentes, es entender que los procesos de salud y enfermedad nacen de las particularidades culturales y territoriales de las poblaciones y que en lugar de establecer una dinámica de intervención para tratar sólo enfermedades emergentes hay que hacerlo mejor para formar "personas verdaderas".

\section{BiBLIOGRAFÍA}

Belaunde, Luisa Elvira. 2005. El recuerdo de Luna: género, sangre y memoria entre los pueblos amazónicos. Fondo Editorial de la Facultad de Ciencias Sociales, UNMS. Centro Amazónico de Antropología y Aplicación Práctica, CAAAP. Lima. 
El cuerpo como unidad biológica y social: Una premisa para la salud sexual y reproductiva

Bertherat, Therese y Carol Bernstein. I979. El cuerpo tiene sus razones. Autocuración y antigimnasia. Ediciones Nacionales Círculo de Lectores. Edinal Ltda. Bogotá.

Camacho Gónzalez, Hugo Armando (comp.). i995. MÀGUTÁ: La Gente Pescada por Yoí. Tercer Mundo Editores. Leticia. ticia.

Esteban, Mariluz. 2004. La antropología del cuerpo. Género, itinerarios corporales, identidad y cambios. Editorial Bellaterra. Barcelona.

Fuentes VÁsquez, Lya Yaneth. 200I. "Representaciones de los cuerpos femenino y masculino, salud y enfermedad. Una revisión de los anuncios publicitarios del Excelsior de I920 a 1990”. En En otras palabras 9:62-85.

Garnelo, Luiza y Esther Jean Langdon. 2005. "A Antropología e A Reformulação das práticas Sanitarias na Atenção Básica a Saúde”. En M. De Souza Minayo, y C. Coimbra Jr. Críticas e Atuantes. Ciencias sociais e humanas em saúde na América Latina. Editora Fiocruz. Río de Janeiro.

Goulard, JeAn-Pierre. 2000. “Los Ticuna”, en F. Santos y F. Barclay (eds.). Guía etnográfica de la Alta Amazonia. Volumen I. IFEA, FLACSO.

2002. "Indios de la frontera, fronteras del indio. Una sociedad indígena en tres Estados-naciones: Los Ticuna”. En Françoise Morin y Roberto Santana. Lo transnacional, instrumento y desafío para los pueblos indígenas. Ediciones Abya-Yala. Quito.

2006. Entre mortales e inmortales. El ser según los Ticuna (Amazonia). Texto inédito.

Hahn, Robert A. y Arthur Kleinman. I983. 'Biomedical Practice and Anthropological Theory: Frameworks and Directions”. Annual Review of Anthropology, I2.

HALBMAYER, ERNST. I997. "La construcción cultural de las relaciones de género entre los Yukpa. La ideología del dominio masculino y el poder de las mujeres”. En M. Perrin, y M. Perruchón. Complementariedad entre hombre y mujer. Relaciones de género desde la perspectiva amerindia. Ediciones Abya-Yala. Quito.

Langdon, Esther JeAn. I995. A doença como Experiência: A construção da doença e seu desafio para a prática médica. Palestra oferecida na conferencia 30 anos Xingu. Escola Paulista de Medicina. Sao Paulo. do século. Trabalho apresentado no V Congreso Latinoamericano de Ciencias Sociales y Medicina. Universidad Federal de Santa Catarina. Isla Margarita. 
Londoño Sulkin, Carlos David. 2004. Muinane: un proyecto moral a perpetuidad. Universidad de Antioquia. Medellín.

Mahecha Rubio, Danny. 2004. La formación de masa goro "personas verdaderas". Pautas de Crianza entre los Macuna del bajo Apaporis. Tesis para aspirar al título de Magíster en Estudios Amazónicos. Universidad Nacional de Colombia. Leticia.

Martin, Emily. 1992. The Woman in the Body. A Cultural Analysis of Reproduction. With a new introduction. Beacon Press. Boston.

Maturana Romesín, Humberto. I993. Desde la biología a la psicología. Editorial Synthesis. Santiago de Chile.

Meloni Vieira, Elisabeth. i999. A medicalizaçao do corpo feminino, en Giffin, Karen y Hawker, Sara. (Organizadoras). Questões da Saúde Reprodutiva, Fundaçao Oswaldo Fiocruz. Río de Janeiro.

Nieto, Juana Valentina. 2006. Mujeres de la abundancia. Tesis presentada para optar por el grado de Magíster en estudios amazónicos. Línea de investigación Historias y Culturas Amazónicas. Universidad Nacional de Colombia. Leticia.

Overing, Joanna y Alan Passes. 200o. "Introduction: Conviviality and the opening up of Amazonian anthropology”. En The Anthropology of Love and Anger: The Aesthetics of conviviality in Native Amazonia. Routledge. Londres.

Reyes, Giovanna. 2008. Analogías y antagonismos en salud sexual y reproductiva entre población Ticuna y los servicios de salud del municipio de Leticia. Tesis presentada para optar por el grado de Magíster en estudios amazónicos. Línea de investigación Desarrollo regional. Universidad Nacional de Colombia. Leticia.

Rodrigues, José Carlos. 2005. “Os corpos na antropología”. En M. De Souza Minayo y C. Jr. Coimbra. Críticas e Atuantes. Ciencias Sociais e humanas em saúde na América Latina. Editora Fiocruz. Río de Janeiro.

Seeger, Anthony, Roberto Da Matta y Eduardo Viveiros de Castro. I987. "A constrüçao da pessoa nas sociedades indígenas brasileiras", en Oliveira João Pacheco de (org.) Sociedades indígenas \& indigenismo no Brasil. Editora UFRJ Marco Zero. Río de Janeiro.

Viveiros de Castro, Eduardo. I979. "A fabricaçao do corpo na sociedade xinguana”. Boletim de Museo Nacional. Nova Serie Antropología. (32). 2002. "Perspectivismo e multinaturalismo na América indígena”. Capítulo 7. En A inconstancia da alma selvagem e outros ensayos de antropología. Editorial Cosac \& Naify. Sao Paulo. 